Rev. Elev. Méd. vét. Pays trop., 1974, 27 (2) : 169-175.

\title{
La fièvre aphteuse en Éthiopie. Distribution des sérotypes de virus aphteux
}

\author{
par J. L. MARTEL (*)
}

\begin{abstract}
RESUME
En faisant la synthèse d'informations d'origines diverses, la présente note donne l'inventaire des types de virus aphteux isolés en Ethiopie de 1957 à 1973.

Les trois types $\mathrm{O}$, $\mathrm{A}$ et $\mathrm{C}$ ont été identifiés, mais aucun virus des types S.A.T. n'a été isolé, bien que ceux-ci existent dans les pays limitrophes.

Le système de transport du bétail sur pied, le long des routes commerciales traditionnelles, contribue largement à la dissémination des virus à l'intérieur du territoire éthiopien.
\end{abstract}

\section{INTRODUCTION}

En 1932, la fièvre aphteuse a été observée en Erythrée (28) et la première déclaration officielle de la maladie remonte, à notre connaissance, à $1953(13,29)$.

De 1957 à 1969, une vingtaine de prélèvements provenant de la région d'Addis-Abeba et d'Asmara ont été adressés au Laboratoire Mondial de Référence pour le typage des virus aphteux de Pirbright. Les typages alors effectués révélèrent la présence des trois types $\mathrm{O}, \mathrm{A}$ et $\mathrm{C}$.

Jusqu'à une période récente, et à l'image de ce qui se passe généralement dans la plupart des pays africains $(1,4,6)$, la fièvre aphteuse a été considérée sans grande importance en Ethiopie où cependant elle sévit souvent de façon enzootique. Il faut reconnaître que la fréquente discrétion de la symptomatologie de la fièvre aphteuse chez le bétail indigène rend le diagnostic souvent difficile et l'attention des

(*) Mission Vétérinaire Française en Ethiopie (I.E.M.V.T.), Service de la Fièvre Aphteuse, Imperial Veterinary Institute, P.O.B. 19, Debre-Zeit, Ethiopie. éleveurs et des autorités sanitaires locales est d'abord attirée par des affections beaucoup plus meurtrières, comme la peste bovine, ou limitant considérablement l'extension de l'élevage, comme les protozooses avec au premier chef les trypanosomoses.

Toutefois, comme l'a souligné J. B. BROOKSBY au dernier Congrès Mondial de Médecine Vétérinaire de Mexico (7), la situation de la fièvre aphteuse en Afrique est unique à deux égards :

1. L'existence de six types viraux sur le continent africain, sur les sept types connus dans le monde;

2. La diversité des hôtes sensibles domestiques et sauvages vivant souvent au contact les uns des autres. Aussi l'existence de cette affection en Afrique, avec ses particularités, est-elle préoccupante à l'échelle internationale.

Dans les pays africains mêmes, l'existence de la fièvre aphteuse devient un problème de plus en plus important au fur et à mesure que les grandes campagnes de prophylaxie contrôlent et éliminent les maladies meurtrières auxquelles nous faisions allusion plus haut. Par ailleurs, 
nous avons pu constater à plusieurs reprises l'importance médicale que pouvait prendre dans certains cas la fièvre aphteuse en Ethiopie, même sur le bétail indigène : avortements, mortalité des veaux, immobilisation des bœufs de commerce présentant de graves lésions podales, imposant parfois l'abattage sur place des malades.

Le développement de l'élevage moderne, particulièrement l'élevage laitier à base de bétail amélioré, replace le problème de la fièvre aphteuse dans le même contexte que dans les pays développés.

Enfin, les exportations de viandes fraîches ou congelées, que l'Ethiopie envisage sur des marchés étrangers très rémunérateurs $(15,17$, $23,24)$, ne pourront être réalisables que dans la mesure où des garanties sanitaires seront présentées aux pays importateurs. Sur ce point particulier, le problème de la fièvre aphteuse est appelé à prendre une des premières places, sinon la première, dans les préoccupations des services sanitaires (27).

C'est dans cette perspective qu'un travail épidémiologique a été entrepris en 1969. Les premiers résultats obtenus en 1971, fruits d'une collaboration étroite entre le Ministère de l'Agriculture du Gouvernement Ethiopien, la Mission Vétérinaire Française en Ethiopie et les Instituts Français de la Fièvre Aphteuse (Institut Mérieux et Laboratoire Roger Bellon), font l'objet d'un rapport à l'Office International des Epizooties (5).

Actuellement, la section des diagnostics du Service de la Fièvre Aphteuse, établi au sein du Laboratoire de la Direction Générale des Services Vétérinaires (Imperial Veterinary Institute) continue ce travail de surveillance permanente de la fièvre aphteuse en Ethiopie (25).

La présente note a pour objet de faire le point sur ce que nous savons de la situation actuelle de la fièvre aphteuse en Ethiopie. Nous donnons l'inventaire des types de virus aphteux isolés, inventaire illustré par une carte de la distribution de ces virus.

\section{MATERIEL ET METHODE}

Les résultats présentés dans cette note portent exclusivement sur des identifications de type effectuées directement à partir des virus isolés des lésions. Nous ne tenons pas compte ici des examens sérologiques, souvent fort utiles pour faire un diagnostic rétrospectif dans un foyer déclaré trop tardivement ou pour faire des sondages dans une région. Ce type de diagnostic indirect n'est valable que dans la mesure où les animaux ne sont pas vaccinés contre la fièvre aphteuse. Or la vaccination antiaphteuse tend à se généraliser dans les fermes laitières et certains ranches.

L'isolement et l'identification des virus est la méthode de choix : depuis la création du Service de la Fièvre Aphteuse à Debré-Zeit, on a pu ainsi conserver au congélateur toute la collection des virus isolés en Ethiopie, en vue de leur étude sérologique et immunologique.

\section{A. PRELEVEMENTS}

Cette méthode suppose des prélèvements correctement pratiqués, à un stade précoce de la phase aiguë de la maladie et soigneusement conservés jusqu'à la réception au laboratoire.

Chaque fois qu'un foyer nous est déclaré, nous nous rendons sur les lieux dans les meilleurs délais. Parfois ce sont les vétérinaires ou les assistants vétérinaires qui rencontrent la maladie à l'occasion de leurs tournées; ils font alors eux-mêmes les prélèvements d'aphte lingual ou podal. Dans ce but, nous leur fournissons des flacons à prélèvements contenant de la glycérine tamponnée et des indications sur la technique à suivre pour la collecte et l'expédition de ces prélèvements (10).

\section{B. ISOLEMENT DES VIRUS APHTEUX}

1. Le matériel virulent est constitué par un broyat d'aphte dans du tampon phosphate chloroformé à 7 p. 1000 : une partie d'aphte pour neuf parties de tampon. Cet extrait est clarifié par centrifugation et inoculé soit à des animaux de laboratoire, soit, le plus souvent, à des cultures cellulaires.

2. L'inoculation sur souriceaux nouveau-nés est effectuée par voie intrapéritonéale $(0,1 \mathrm{ml})$. La multiplication du virus entraîne la mort des souriceaux dans un délai de 24 heures à 8 10 jours. 
3. L'inoculation du cobaye par voie intradermo-plantaire aux membres postérieurs ( $0,5 \mathrm{ml}$ par coussinet plantaire) fait apparaitre un aphte primaire au point d'inoculation entre 24 et 48 heures. Les aphtes primaires sont récoltés par arrachage avec une pince stérile.

4. L'inoculation de cultures cellulaires est la technique la plus utilisée à Debré-Zeit : cellules primaires de rein de veau, cellule de lignée continue (BHK 21 et IBR S2) cultivées en couches monocellulaires en tubes.

On prépare des dilutions au $1 / 10,1 / 100$ et $1 / 1000$ du broyat d'aphte et on inocule 5 tubes de culture cellulaire par dilution de matériel virulent. La multiplication du virus aphteux se manifeste sur ces cellules par l'apparition d'un effet cytopathogène du type Entérovirus entre 16 et 24 heures. En général, on pratique deux à trois passages sur culture cellulaire avant de procéder à l'identification sérologique du virus.

\section{IDENTIFICATION SEROLOGIQUE DES VIRUS}

\section{Les éléments de la réaction}

a) Le sérum hémolytique de lapin anti-hématies de mouton est celui du commerce (Institut Pasteur de Paris).

b) Les hématies proviennent des moutons locaux.

c) Le complément utilisé est du complément lyophilisé du commerce (Institut Pasteur de Paris, Institut Mérieux, Laboratoire Roger Bellon).

d) Les premiers sérums de référence ont été fournis par le Laboratoire Roger Bellon pour les types européens $\mathrm{O}, \mathrm{A}$ et $\mathrm{C}$. Le laboratoire Mondial de Référence de Pirbright nous a fourni les sérums vis-à-vis des trois types africains S.A.T.

Actuellement, nous produisons à Debré-Zeit des sérums hyperimmuns sur cobayes à partir des souches de virus aphteux isolés localement. Les cobayes, inoculés par voie intradermo-plantaire avec un virus aphteux adapté après six à sept passages sur cobayes, doivent présenter une fièvre aphteuse expérimentale caractérisée par l'apparition d'un aphte primaire au point d'inoculation en 24 heures et des aphtes secondaires de généralisation en 48 heures. Deux à trois mois après la guérison (8) ces animaux reçoivent une injection de rappel, par voie intramusculaire, avec le même virus additionné de saponine. Ils ne doivent alors pas présenter de signes de fièvre aphteuse. Dix jours plus tard, les cobayes sont saignés et les sérums récoltés individuellement. Le titre et la spécificité de type de chaque sérum sont contrôlés avant d'être mélangés pour constituer un lot de sérum hyperimmun.

e) Les antigènes à identifier sont constitués par l'extrait en tampon phosphate chloroformé soit des aphtes bovins, soit des souriceaux nouveau-nés morts après l'inoculation, soit des aphtes expérimentaux de cobayes, ou bien par le milieu des cultures de cellules présentant un effet cytopathogène 24 heures après l'inoculation.

\section{La réaction d'identification}

Nous utilisons la réaction de fixation du complément, technique de Kolmer à 100 p. 100 d'hémolyse, en tubes, dans laquelle le complément entre à raison de deux unités hémolytiques 100 p. 100 , le sérum hémolytique à raison de deux unités hémolytiques et les hématies de mouton à raison de 1,15 p. 100 du culot globulaire.

Lorsque l'antigène se révèle anticomplémentaire, on prépare des dilutions en progression géométrique de raison 2 jusqu'au 1/64. La réaction de fixation du complément est alors pratiquée sur chacune de ces dilutions d'antigène.

\section{RESULTATS ET DISCUSSION}

\section{A. DISTRIBUTION CHRONOLOGIQUE}

Depuis 1957, date des premiers typages de virus aphteux d'origine éthiopienne, on peut considérer que les recherches sur la fièvre aphteuse ont évolué en trois phases :

\section{Période préliminaire : $1957-1969$}

L'Ethiopie ne possède pas encore de laboratoire capable d'effectuer l'isolement, l'identification et le typage des virus aphteux. 
Pendant cette période de douze années, une vingtaine de prélèvements ont été occasionnellement envoyés à Pirbright (prélèvements $\mathrm{n}^{\mathrm{os}} 1$ à 20). Le type $O$ prédomine très nettement : sur vingt résultats positifs, 18 le sont pour le type $O$, un seul cas est positif pour le type $A$ (en 1957) et un seul cas est positif pour le type C (en 1957 également). De 1958 à 1969, seul le type $\mathrm{O}$ a été identifié par le Laboratoire Mondial de Référence de Pirbright (22). Nous ne disposons pas de commémoratifs précis sur ces prélèvements.

\section{Début des études systématiques : 1969 - $1^{\text {er }}$ semestre 1971}

En 1969, les premiers typages de virus aphteux ont pu être réalisés en Ethiopie, à l'Imperial Veterinary Institute.

Une équipe spécialisée s'est rendue sur le terrain pour informer les services vétérinaires des différentes provinces de la conduite à tenir en cas d'épidémie de fièvre aphteuse et fournir du matériel de prélèvement.

Pendant cette période de démarrage, le laboratoire de Debré-Zeit a pu recevoir des échantillons de virus aphteux de différentes provinces. Dix-neuf prélèvements effectués sur des bovins (du $n^{\circ} 21$ au $n^{\circ} 39$ ) ont été positifs : huit pour le type $\mathrm{O}$ et onze pour le type $\mathrm{A}$.

Les travaux réalisés pendant cette période font l'objet d'un rapport publié en 1972 (5). Notons simplement que les isolements de type $\mathrm{A}$ ont été très importants et que, par contre, aucun virus de type C n'a été isolé.

\section{Période actuelle : 20 trimestre 1971- 1973}

Actuellement, le Service de la Fièvre Aphteuse installé à l'Imperial Veterinary Institute reçoit tous les prélèvements effectués dans les foyers de fièvre aphteuse déclarés en Ethiopie. Seuls les examens pratiqués pendant cette période constituent des observations personnelles de l'auteur de cette note.

Au 31 décembre 1973, 59 prélèvements ont été positifs (prélèvements $\mathrm{n}^{\text {10s }} 40$ à 98 ) : 36 le sont pour le type $O$ et 23 pour le type C. Remarquons qu'aucun virus de type A n'a été isolé depuis $1971\left(^{*}\right)$. Par contre le type $C$, qui a recommencé à se manifester en 1971, a été largement retrouvé dans les foyers étudiés en 1972 et 1973 . Tous ces prélèvements ont été réalisés sur des bovins (taurins ou zébus) à l'exception d'un seul (prélèvement $n^{\circ} 83$ ) pratiqué sur des porcs.

$$
\text { TABLEAU } \mathrm{N}^{\circ} \mathrm{I}
$$

Distribution chronologique des types de virus aphteux - Isolés en Ethiopie

\begin{tabular}{|c|r|r|r|c|}
\hline Përiode & Type 0 & Type A & Type C & $\begin{array}{l}\text { Tota1 des } \\
\text { virus typés }\end{array}$ \\
\hline $1957-1969$ & 18 & 1 & 1 & 20 \\
$1969-1971$ & 8 & 11 & - & 19 \\
$1971-1973$ & 36 & - & 23 & 59 \\
\hline Total & 62 & 12 & 24 & 98 \\
\hline
\end{tabular}

L'ensemble de ces informations (cf. tableau) montre :

1. La permanence et l'importance des foyers de type 0 . Cette constatation coïncide avec la présence de ce même type $O$ signalée dans tous les pays limitrophes de l'Ethiopie: Soudan, Kenya et Somalie (16);

2. L'apparition, semble-t-il cyclique, des épizooties de type $\mathrm{A}$ et $\mathrm{C}$;

3. L'absence d'isolement de virus de type S.A.T., bien que ces virus soient signalés dans certains pays limitrophes: S.A.T. 1 au Kenya et au Soudan, S.A.T. 2 au Kénya (16).

On peut penser que l'Ethiopie est naturellement protégée contre l'invasion des virus S.A.T. existant dans les pays voisins car:

- L'Ethiopie est traditionnellement un pays exportateur de bétail. Les tendances commerciales ne sont donc pas favorables à l'importation accidentelle en Ethiopie de virus étrangers par les troupeaux de commerce;

- Les zones frontalières du territoire éthiopien ont une altitude généralement inférieure à 1500 mètres, favorable aux glossines $(2,3)$. Les trypanosomoses constituent dans ces zones un important obstacle pour l'élevage qui est particulièrement clairsemé à l'ouest et au sudouest, le long de la frontière du Soudan. Ces zones peuvent ainsi être considérées comme une barrière naturelle pour les virus étrangers.

(*) Depuis la rédaction de cet article, un virus de type A a été isolé en février 1974. 


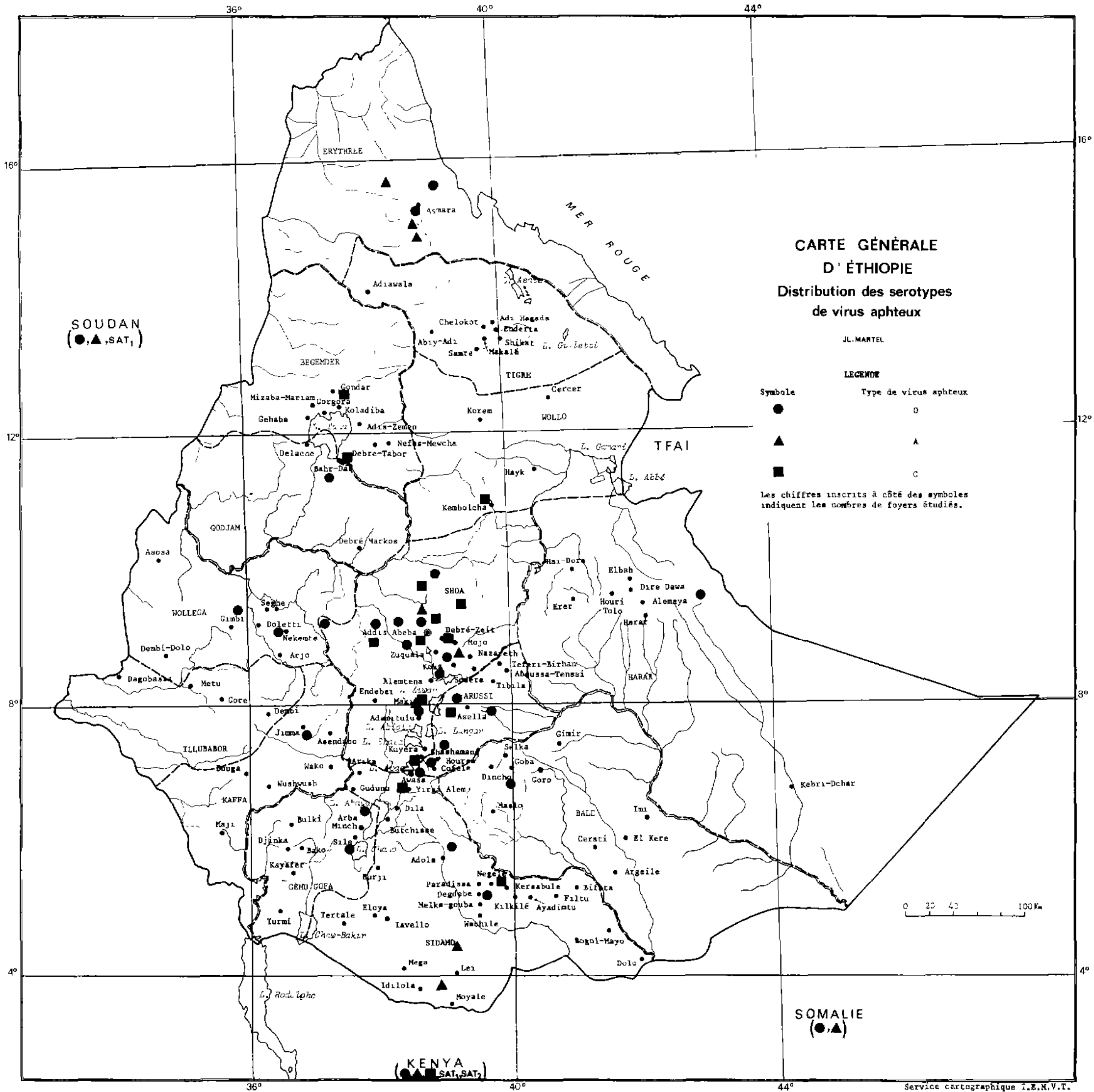


Toutefois cette protection naturelle reste très relative :

- Un nouveau courant moderne d'importation de bétail amélioré s'amorce, notamment dans le cadre du projet de développement de l'élevage laitier de la région d'Addis-Abeba. Tout contrôle sanitaire à l'importation doit être très sévère si l'on veut protéger le territoire éthiopien contre l'introduction de virus aphteux étrangers;

- Par ailleurs, si les zones frontalières avec le Soudan ne sont pas actuellement favorables au transport des virus aphteux par les animaux domestiques, trop rares, un risque persiste, matérialisé par la présence de la faune sauvage, réservoir potentiel de virus aphteux $(9,11,19$, $18,20,21)$.

- Quand au sud, signalons qu'il existe de petits mouvements frontaliers de troupeaux d'élevage entre Dolo et Lei, près de la frontière du Kenya. Enfin, lorsque la route asphaltée Addis-Abeba - Nairobi, actuellement en cours de construction, sera ouverte au trafic routier intense et rapide une surveillance sanitaire s'imposera.

\section{B. DISTRIBUTION GEOGRAPHIQUE}

Les prélèvements qui ont fait l'objet de typage proviennent pratiquement de toutes les régions d'Ethiopie; nous manquons d'informations dans deux provinces seulement : celle du Tigré au nord et de l'Illubabor à l'extrême ouest du territoire éthiopien.

La répartition des foyers n'est pas homogène, et bien souvent les foyers étudiés sont situés près des axes de communication. Certes l'infrastructure routière est favorable à la transmission des informations et aux expéditions de prélèvements. Aussi n'avons-nous pas la prétention de présenter ici l'inventaire exhaustif des virus responsables de toutes les épizooties ayant sévi en Ethiopie; les difficultés de déplacement et la faible densité du personnel vétérinaire dans certaines régions limitent considérablement les informations.

Mais nous pensons que la concentration des points de prélèvements dans certaines zones peut être aussi le reflet de conditions épizootiologiques particulièrement favorables au virus aphteux. Les troupeaux de commerce se déplacent le plus généralement à pied : les contacts ayec les animaux des régions traversées étant inévitables, ces mouvements commerciaux sont très favorables à la dissémination des virus le long des pistes suivies.

Certains axes sont très fréquentés, telle la Vallée du Rift qui draine le bétail de l'importante région d'élevage que représente la province méridionale du Sidamo, vers d'une part l'abattoir industriel situé près de Shashemene, au carrefour des routes du Gému Goffa, du Sidamo et du Balé, et d'autre part vers l'agglomération d'Addis-Abeba plus au nord. C'est ainsi que de nombreux foyers de fièvre aphteuse ont été déclarés et étudiés le long de cette piste et à son aboutissement à Addis-Abeba. Il est à ce sujet intéressant de remarquer que très souvent les premiers foyers nous sont signalés dans des exploitations possédant des animaux améliorés (fermes laitières, porcheries industrielles). En réalité ces fermes ne sont pas à l'origine de la maladie, mais constituent des révélateurs de l'infection du simple fait de la sensibilité particulière des animaux de ces exploitations au virus aphteux, lequel a été souvent véhiculé à bas bruit par les troupeaux de commerce depuis les zones d'élevage extensif où la maladie est restée inconnue, faute d'examen sanitaire attentif.

\section{CONCLUSION}

Seuls les types $\mathrm{O}, \mathrm{A}$ et $\mathrm{C}$ ont été isolés et identifiés en Ethiopie sur une période de quinze années d'observation portant sur 98 prélèvements positifs.

Le type $O$ sévit à l'état enzootique et en permanence dans l'ensemble du territoire éthiopien. Cela correspond à la présence de ce type signalé dans tous les pays limitrophes: Soudan, Kenya, Somalie.

Par contre, l'apparition des types A et $\mathrm{C}$ semble être cyclique.

L'absence d'isolement des types S.A.T. contraste avec la présence du type S.A.T. 1 au Kenya et au Soudan, et du type S.A.T. 2 au 
Kenya. On peut penser que l'Ethiopie est naturellement protégée contre l'invasion par les virus existant dans les pays limitrophes. Mais les facteurs responsables de cette situation relativement favorable peuvent évoluer : le contrôle sanitaire des importations de bétail doit être sévère et la surveillance des foyers de fièvre aphteuse doit être soutenue et autant que possible accrue notamment dans les régions frontalières.

\section{SUMMARY}

Foot and mouth disease in Ethiopia.

Distribution of foot and mouth disease virus serotypes

The present note, gathering informations of various sources, gives the list of foot and mouth disease virus types isolated in Ethiopia between 1957 and 1973.

The three types $O, A$ and $C$ have been identified, but no SAT type virus has been isolated although these do exist in the neighbouring countries.

The cattle movements on foot along the traditional trade routes widely contribute to the dissemination of viruses inside the ethiopian territory.

\section{RESUMEN}

La fiebre aftosa en Etiopia.

\section{Distribucion de los serotipos de virus aftoso}

Haciendo la sintesis de informaciones de varias origenes, la presente nota de la lista de los tipos de virus de fiebre aftosa aislados en Etiopia de 1957 a 1973.

Los tres serotipos $\mathrm{O}, \mathrm{A}$ y C fueron identificados, pero no se aisló ningùn virus de los tipos SAT a pesar de que existan estos tipos en los paises vecinos.

El sistema de transporte del ganado de pie, a lo largo de las rutas comerciales tradiciónales contribuye mucho en la diseminación de virus al interior del teritorio etiopiano.

\section{BIBLIOGRAPHIE}

1. ATANG (P. G.). Foot and mouth disease in Africa. Bull. epiz. Dis. Afr., 1968, 16: 129-137.

2. BALIS (J.), BERGEON (P.). Etude de la répartition des glossines en Ethiopie. Bull. Org. mond. Santé, 1968, 38: 809-813.

3. BALIS (J.), BERGEON (P.). Etude sommaire de la répartition des glossines dans l'Empire d'Ethiopie. Rev. Elev. Méd. vét. Pays trop., 1970, 23 (2) : 181-187.

4. BEATON (W. G.). L'épizootologie régionale comparée de la fièvre aphteuse (Afrique au sud du Sahara). Bull. Off. int. Epiz., 1959, 50: 473-481.

5. BERSON (J.P.), COLSON (X.), FIKRE (J.), VIGIER (M.), ASSEFA (W. G.), GUERCHE (J.), BLANC (R.), PRUNET (P.), Etude épizootologique de la fièvre aphteuse en Ethiopie (1969-1971). Bull. Off. int. Epiz. 1972, 77 (3-4) : 395-620.

6. BROOKSBY (J.B.). Epizootiology of foot and mouth disease in developing countries. World Anim. Rev. F.A.O., 19721 : 10-13.

7. BROOKSBY (J.B.). Foot and mouth disease as a world problem and measures for its control in different regions : summary of situation in Africa. Proc. 19th World Veterinary Congress, Mexico, 1971, 3 : 1202-1207.

8. BROOKSBY (J. B.). The technique of complement fixation in foot and mouth disease $\mathrm{Re}$ search. London, Her Majesty's Stationnery Office, 1952, (Agricultural Research Council Report Series, no 12).
9. BROOKSBY (J.B.). Wild animals and the epizootiology of foot and mouth disease. Symp. zool. Soc. Lond., 1968, 24 : 1-11.

10. BROOKSBY (J. B.), DAVIE (J.), HEDGER (R.S.). Transmission of virus samples. Bull. Off. int. Epiz., 1964, 61 (11-12) : 1605-1615.

11. CONDY (J.B.), HERNIMAN (K. A.J.), HEDGER (R. S.). Foot and mouth disease in wildlife in Rhodesia and other African territories. $J$. comp. Path. Ther., 1969, 79: 27-31.

12. DURAUD (P.). Report to the Government of Ethiopia on the control of animal diseases. Rome, F.A.O., 1958. (Report F.A.O., n 905.)

13. ERYTHREAN GOVERNMENT - Monthly Veterinary Bulletin, January 1949 - September 1961.

14. FALCONER (J.). The epizootiology and control of foot and mouth disease in Botswana. Vet. Rec., 1972, 91 (15) : 354-359.

15. F.A.O. - Livestock and meat industries in Ethiopia. Present and future. Rome, F.A.O., 1973.

16. F.A.O. - W.H.O. - O.I.E. - Annuaire de la Santé Animale. Rome, F.A.O., 1971.

17. GUIORGUIS (A.W.), HOLLYER (J.A.), IMAN (M.). Marketing prospects for Ethiopian livestock and livestock products in the Near and Middle East. Addis-Ababa, Addis-Ababa Livestock and Meat Board, 1972.

18. HEDGER (R.S.). Foot and mouth disease and the african buffalo (Syncerus caffer). J. comp. Path. Ther. 1972, $82: 19-28$. 
19. HEDGER (R. S.), CONDY (J. B.), FALCONER (J.). The isolation of foot and mouth disease virus from african buffalo (Syncerus caffer). Vet. Rec., $1969,84(20)$ : 516-517.

20. HEDGER (R.S.), CONDY (J.B.), GOLDING (S. M.). Infection of some species of african wildlife with foot and mouth disease virus. J. comp. Path. Ther. 1972, 82 : 455-461.

21. HEDGER (R.S.), FORMAN (A. J.), WOODFORD (M. H.). Le virus de la fièvre aphteuse chez le buffle est-africain. Bull. epiz. Dis. Afr., 1973, 21 : 101-103.

22. HOBDAY (J.). Communication personnelle.

23. IMPERIAL ETHIOPLAN GOVERNMENT Livestock and Meat Board. Livestock and products marketing project. Addis-Ababa, Livestock and Meat Board, 1971.

24. IMPERIAL ETHIOPIAN GOVERNMENT Livestock and Meat Board. Provincial livestock development studies. Addis-Abeba, Livestock and Meat Board, 1970-1972.
25. IMPERIAL ETHIOPIAN GOVERNMENT The department of Veterinary Service, Ministry of Agriculture. A review of animal health and livestock productivity factors in Ethiopia: 19651971.

26. LIBEAU (J.). Situation actuelle de la fièvre aphteuse en Afrique au Sud du Sahara. Bull. Off. int. Epiz., 1960, 54: 74-89.

27. O.I.E. - F.A.O. - Réunion d'un groupe de travail O.I.E.-F.A.O. pour examiner les critères régissant l'importation de bovins de boucherie et de viandes à partir de pays non complètement indemnes de maladies à virus exotiques pour l'Europe en vue de faciliter le commerce interrégional. Bull. Off. int. Epiz., 197277 (1-2): 293307.

28. PIRANI (A.): in CURASSON (G.). Traité de pathologie exotique vétérinaire et comparée. Tome I, Paris, Vigot Frères, 1942.

29. VILLON (A.). Epizootologie de la fièvre aphteuse en Ethiopie. Identification des types de virus. Thèse Méd. vét. Lyon, 1973, nº 51. 